\title{
Molecular diversity in venom proteins of the Russell's viper (Daboia russellii russellii) and the Indian cobra (Naja naja) in Sri Lanka
}

\author{
Mieko Suzuki $^{1}$, Takeshi Itoh $^{1}$, B. M. Anuruddhe I. K. Bandaranayake ${ }^{2}$, J. G. Shirani Ranasinghe ${ }^{2}$, Seranath \\ B.P. Athauda ${ }^{2}$ and Akihiko Moriyama ${ }^{1}$ \\ ${ }^{1}$ Division of Biomolecular Science, Graduate School of Natural Sciences, Nagoya City University, Mizuho, Nagoya 467-8501, Japan; \\ and ${ }^{2}$ Department of Biochemistry, Peradeniya University, Peradeniya, Sri Lanka
}

(Received 23 November 2009; and accepted 23 December 2009)

\begin{abstract}
To examine the molecular diversity of the venom proteins of the Russell's viper (Daboia russellii russellii) and the Indian cobra (Naja naja) in Sri Lanka, we isolated 38 venom proteins through a combination of anion exchange chromatography followed by reversed-phase high performance liquid chromatography. From the venom of D. r. russellii we isolated 15 proteins: 5 isozymes of phospholipase $\mathrm{A}_{2}\left(\mathrm{PLA}_{2}\right), 4$ serine proteases, 2 C-type lectin-like proteins, 2 L-amino acid oxidases, 1 cysteine-rich secretory protein (CRISP), and 1 metalloproteinase. From the venom of N. naja we isolated 23 proteins: 10 isoforms of cytotoxins (CTX), 7 PLA $_{2}$ isozymes, 2 muscarinic toxinlike proteins, 2 CRISPs, 1 nerve growth factor, and 1 new thrombin-like serine protease. Most of these proteins contained new amino acid sequences for each species, indicating molecular diversity in venom proteins. The entire amino acid sequences of $\mathrm{PLA}_{2} 3$ from D. r. russellii and CTX7 from $N$. naja were determined. Additionally, the polymorphic amino acid residues of $\mathrm{PLA}_{2} 3$ were preferentially localized on the potential antigenic sites. While 2 types of $\mathrm{PLA}_{2}$ ( $\mathrm{N}$ and $\mathrm{S}$ types) were found in D. r. russellii (India) and D. r. siamensis (Java), all the $\mathrm{PLA}_{2} \mathrm{~s}$ from D. r. siamensis (Burma) were $\mathrm{N}$ type, and those from D. r. russellii (Sri Lanka) were primarily $\mathrm{S}$ type.
\end{abstract}

There are approximately 600 venomous species of snakes in the world. Although there are no accurate figures for the incidence of snakebites, at least 421,000 envenomings and 20,000 deaths are estimated to occur every year according to publications on snakebite and the World Health Organization (WHO) mortality database in 2008 (14). Snakebites caused by the families of Viperidae and Elapidae are particularly dangerous to humans. Not all the snakebites are fatal, yet they may result in the following conditions: permanent physical disability, including limb amputation; chronic ulceration; os-

Address correspondence to: Mieko Suzuki Division of Biomolecular Chemistry, Graduate School of Natural Sciences, Nagoya City University, Mizuho, Nagoya, 467-8501, Japan

Tel: +81-52-872-5851, Fax: +81-52-872-5857

E-mail:m.suzuki@nsc.nagoya-cu.ac.jp teomyelitis with malignant transformation; chronic renal failure; chronic pituitary-adrenal insufficiency;

To avoid confusion in terms of taxonomy, the following names were used in this paper: D. russellii russellii (Sri Lanka), a subspecies of D. russellii found in Sri Lanka and South India, corresponds to D. r. puluchella in the previous taxonomy; $D$. $r$. russellii (India), a subspecies of D. russellii found in North India, includes D. r. nordicus in the previous taxonomy; D. r. siamensis (Burma), a subspecies of $D$. russellii found in Burma, Thailand, Cambodia and Southern China, includes a large portion of $D$. r. siamensis in the previous taxonomy; D. r. siamensis (Java), a subspecies of D. russellii found in Java, Komodo, Flores and Lomblen, corresponds to D. r. limitis in the previous taxonomy ; D. r. siamensis (Taiwan), a subspecies of D. r. siamensis found in Taiwan, includes $D$. r. formosensis in the previous taxonomy; $N$. naja (Sri Lanka), which was previously classified as $N$. naja polyocellata or $N$. polyocellata. 
and neurological sequelae from intracranial hemorrhages and thromboses.

Sri Lanka is one of the highest-risk countries in terms of snakebite fatality (4) with about 37,000 people suffering from snakebite every year (5). Much of the morbidity and about $95 \%$ of the snakebite mortality in Sri Lanka is due to bites from highly venomous snakes, such as the Russell's viper (Daboia russellii russellii), the hump-nosed viper (Hypnale hypnale), the Indian cobra (Naja naja), and the Indian krait (Bungarus caeruleus) (13). The former two belong to the Vipridae and the latter two belong to the Elapidae families.

Due to variation in the toxin composition of venoms, effective antivenom therapy is a key to the medical management of snakebite. Unfortunately, the use of antivenom serum (AVS) made in different regions has limited or no efficacy against non-local snake species, suggesting extreme variation in venom composition. For example, Haffkine polyspecific AVS (Haffkine Laboratories, Mumbai, India) adapted to Indian species has been used for the treatment of envenoming, and occasionally found to be ineffective in Sri Lanka $(3,12,17,22,24,26)$. It is also reported that Burmese antivenom serum did not neutralize the hemolytic activity of the venom of $D$. r. russellii (Sri Lanka) in vitro, and the relative content of toxins was different in each local snake (28).

Russell's viper is classified into 2 subspecies, namely $D . r$ r russellii and $D$. r. siamensis (18). The former was previously divided into 3 subspecies, namely D. r. russellii (India, Pakistan and Bangladesh), D. r. pulchella (Sri Lanka) and D. r. nordicus (Northern India), while the latter previously included 3 subspecies, namely D. r. siamensis (Burma, Thailand, Cambodia and Southern China), D. r. limitis (Java, Komodo, Flores and Lomblen) and D. $r$. formosensis (Taiwan). Similarly, the taxonomy of the Asiatic cobra has been changed several times. At present, there are 14 species of Asiatic cobra, which belong to the genus Naja, although most of them were previously classified as subspecies of Naja naja. Indian cobra living in the Indian subcontinent and in Sri Lanka, which were previously classified as $N$. n. naja and $N$. n. polyocellata, respectively, have been combined into the single species $N$. naja (29).

These historical changes in the taxonomy of venomous snakes suggest that these venomous snakes are on their way to differentiation into separate subspecies based on their geographic origin, which is resulting in diversification in the antigenicity of their venom proteins. Furthermore, phospholipases
$\mathrm{A}_{2}\left(\mathrm{PLA}_{2} \mathrm{~s}\right)$ and cytotoxins (CTXs) are the major components in the venom of the Russell's viper and the Indian cobra, respectively. Both are known to be encoded by multigene families that have evolved through a process of gene duplication followed by accelerated evolution in the protein coding region $(7$, $8,19,20)$. Therefore, they make a family of toxins with a highly conserved structural fold but widely varying surface amino acid residues. The differences in the primary structure of these and other venom proteins may directly influence the effectiveness of AVS.

The current study focused on the proteomic analyses of the venoms of the Russell's viper (D. russellii) and the Indian cobra (N. naja) living in Sri Lanka and compared them with the primary structures of the venom proteins from the same species living in other locales.

\section{MATERIALS AND METHODS}

Venoms. Venoms used for the present study were collected from the Russell's viper and the Indian cobra which were caught in Sri Lanka and kept in Peradeniya University. The venoms were lyophilized and stored in a freezer until use.

Two-dimensional chromatography. A lyophilized sample was dissolved in an original volume of distilled water and was applied onto a column of PA-SP $(8 \times 100 \mathrm{~mm}$; Shimadzu, Kyoto, Japan $)$ that had been equilibrated with $20 \mathrm{mM}$ sodium acetate buffer ( $\mathrm{pH}$ 5.5). After being washed with the same buffer, proteins were eluted with a linear $\mathrm{NaCl}$ gradient $(0-1 \mathrm{M})$ in the same buffer with a flow rate of $1 \mathrm{~mL} / \mathrm{min}$. One $\mathrm{mL}$ of each fraction was collected. Every two fractions were combined, acidified with the addition of $0.2 \mathrm{~mL}$ of $10 \%$ trifluoroacetic acid (TFA), and applied on a column of Develosil 300 C4-HG5 (4.6 × $150 \mathrm{~mm}$; Nomura Chem., Seto, Japan) equilibrated with $0.1 \%$ TFA. After being washed with $0.1 \%$ TFA, proteins were eluted with a TFA gradient $(0.1 \%-0.1 \% / 60 \%$ acetonitrile $)$ in a total volume of $60 \mathrm{~mL}$. The flow rate was 1.0 and $0.5 \mathrm{~mL} / \mathrm{min}$ for Russell's viper venom and the Indian cobra venom, respectively. A 1-mL sample of each fraction was collected and concentrated in a vacuum concentrator.

Amino acid sequence determination. Amino-terminal amino acid sequences were determined by an automated protein sequencer (Model 491 cLC Protein Sequencer; Applied Biosystems, Foster City, CA, 
USA). To determine the entire primary sequences of proteins, samples were reduced with dithiothreitol, and S-pyridylethylated by the addition of 4-vinylpyridine under vacuum, according to the method outlined by Friedman (6), then digested with trypsin, AspN, or LysC. The digestion was carried out at $37^{\circ} \mathrm{C}$ for $18 \mathrm{~h}$. The digestion was acidified with $10 \%$ TFA and applied onto a column of CAPCELL PAK C-18 (4.6 × $250 \mathrm{~mm}$; Shiseido, Tokyo, Japan). Peptide fragments were eluted with a linear acetonitrile gradient (0 to $60 \%$ ) for $120 \mathrm{~min}$.

Assay for thrombin activity. Thrombin activity was measured using t-butyloxycarbonyl-[(2S)-2-amino3-(benzyloxycarbonyl)propionyl]-prolyl-arginine4-methylcoumaryl-7-amide (Boc-Asp(oBzl)-ProArg-MCA), as a substrate. The reaction mixture, consisting of $5 \mu \mathrm{M}$ Boc-Asp-Pro-Arg-MCA, $0.1 \mathrm{M}$ sodium phosphate buffer $(\mathrm{pH}$ 7.4) and an appropriate amount of enzyme, was incubated at $37^{\circ} \mathrm{C}$ for
$10 \mathrm{~min}$. The reaction was stopped by the addition of a $2.5 \mathrm{~mL}$ of $1.2 \mathrm{M}$ monochloroacetic acid solution. An increase in the fluorescence intensity of the reaction mixture at $460 \mathrm{~nm}$ was measured using an excitation wavelength of $380 \mathrm{~nm}$ (15).

\section{RESULTS}

\section{Isolation of venom proteins of the Russell's viper and the Indian cobra}

To isolate the venom proteins of the Russell's viper and the Indian cobra, venoms were subjected to two-dimensional chromatography on a cation exchange column and then on a reverse phase column. The elution profiles are shown in Fig. 1.

The solution of each peak fraction was subjected to amino-terminal amino acid sequence determination. As a result, 15 and 23 kinds of proteins were identified in the elution of the venoms of $D . r$ r russellii and N. naja, respectively, by applying Blast
A

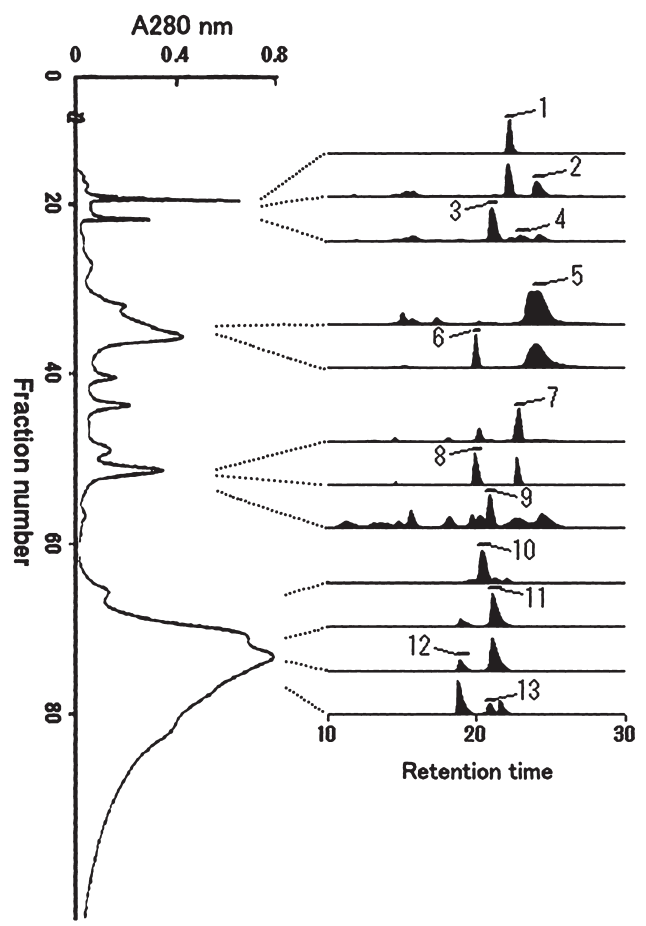

B

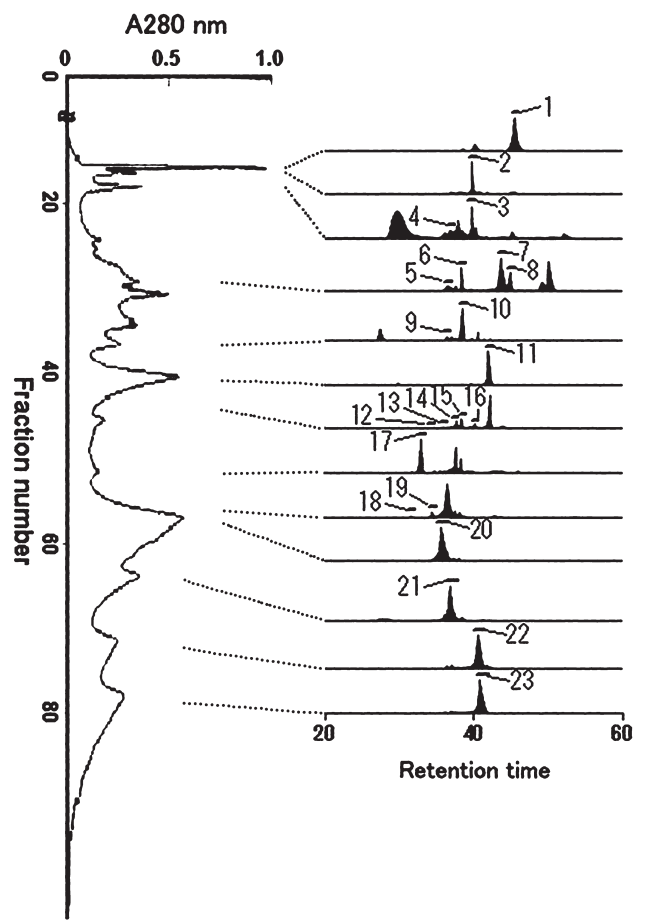

Fig. 1 Purification of the snake venom proteins. (A) Two-dimensional chromatographies of the venom of the Russell's viper, D. r. russellii (Sri Lanka). The venom was applied onto a PA-SP column $(8 \times 100 \mathrm{~mm})$ equilibrated with a $20 \mathrm{mM}$ sodium acetate buffer $(\mathrm{pH} 5.5)$ and was eluted with a linear $\mathrm{NaCl}$ gradient $(0-1 \mathrm{M})$ (Elution profile shown on the left). Each eluted fraction was then applied to the C4 column (DEVELOSIL 300C4-HG-5, $4.6 \mathrm{~mm} \times 150 \mathrm{~mm}$ ) equilibrated with $0.1 \%$ TFA and eluted with a linear acetonitrile gradient (Elution profiles shown on the right). The identified peaks were labeled with numbers according to the order of the elution. (B) Two-dimensional chromatographies of the venom of the Indian cobra, N. naja (Sri Lanka). Chromatographic conditions were the same as the above except that the flow rate for RP-HPLC was half that of $(A)$. The identified peaks were labeled with numbers according to the order of the elution. 
analyses of known proteins. The identified peaks tentatively numbered in the order of the elution are summarized in Table 1 and Table 2. Both the peak 5 and the peak 7 in Fig. 1A were composed of a major protein with a minor homologous protein.

Venom proteins of D. r. russellii

From the peaks of $6,9,11,12$ and 13 of D. r. russellii, 5 isozymes of $\mathrm{PLA}_{2} 1$ to $\mathrm{PLA}_{2} 5$ were purified. Only $\mathrm{PLA}_{2} 1$ belonged to the $\mathrm{N}$ type, which has $\mathrm{Asn}^{1}$ at the amino terminus. On the other hand, the other $4 \mathrm{PLA}_{2}$ isozymes were of the $\mathrm{S}$ type which is characterized by $\mathrm{Ser}^{1}$ at the amino terminus. Among the $\mathrm{S}$ type, $\mathrm{PLA}_{2} 1, \mathrm{PLA}_{2} 2, \mathrm{PLA}_{2} 3$ and $\mathrm{PLA}_{2} 5$ were found to have the same amino-terminal 24 amino acid sequences, and the amino-terminal sequence of $\mathrm{PLA}_{2} 4$ was identical to that of the reported sequence for the $\mathrm{PLA}_{2}$ of D. r. russellii. The peak 1, peak 3, peak 4 and peak 10 appeared to be members of the Factor $\mathrm{V}$ activating proteinase (RVV-V) family, and were tentatively named RVV-V1 to RVV-V4, because of their homology to Vipera russelli proteinase RVV-V alpha (P18964) and Vipera russellii proteinase RVV-V gamma (P18965), respectively. With respect to the amino-terminal 15 amino acid residues, three of four RVV-Vs $(\mathrm{RVV}-\mathrm{V} 1 \sim \mathrm{V} 3)$ had unique sequences for $D$. russellii.

Peak 7-1 and peak 7-2 were identified as C-type lectin-like proteins (CLPs), on the basis of their sequence homology to CLPs of D. r. siamensis, and were designated as CLP1 and CLP2. CLP2 was homologous to CLP5 (Q4PRC8) and CLP7 (Q4PRC6) of D. r. siamensis with $89.5 \%$ homology in the amino-terminal 19 amino acid sequences. $\mathrm{Cys}^{4}, \mathrm{Cys}^{15}$, Tyr $^{16}$ and Phe $^{20}$ were conserved in all CLPs of $D$. russellii. As the peaks 5-1 and 5-2 have a sequence similar to those of the L-amino acid oxidase (LAO) of the Levantine viper (Macrovipera lebetina) (P81375) and the common viper (Vipera berus berus) (P0C2D7), they were classified as members of the LAO family. Peak 2 was identified as a Factor-X activating enzyme (RVV-X) because of its sequence homology to the RVV-X heavy chain of $D$. r. siamensis (Q7LZ61). Peak 10 was tentatively classified as a member of the cysteine-rich secretory protein (CRISP) family, as its partial sequence was identical to that of the Piscivorin precursor (Q7ZTA0) of Agkistrodon piscivorus piscivorus and the Catrin-1/2 precursor (Q7ZT99) of Crotalus atrox, the proteins of which both belong to the CRISP family. This was the first time for these particular LAO and CRISP proteins to be found in the venom of $D$. russellii.
Venom proteins of $N$. naja

Judging from the amino-terminal amino acid sequences, 10 CTXs, 7 PLA $_{2}$ s, 2 kinds of CRISPs, 2 muscarinic toxin-like proteins (MTLPs), a nerve growth factor (NGF) and 1 thrombin-like serine protease (TLP) were found in the venom of the Indian cobra.

Ten peaks (peak 10, peak 11 and peak 16 to peak 23) were identified as CTXs because of their sequence homology to cobra CTXs, and were designated as CTX1 to CTX10 as shown in Table 2. Partial amino acid sequences of CTX4, 5 and 6 were identical to the reported sequence of CTX-1 (P01447), and CTX9 was identical to CTX-2 (P01440) of N. naja (India). The other 6 sequences were novel for $N$. naja CTX. CTX1 and CTX2 were especially unique as they contained $\mathrm{Gln}^{2}, \mathrm{Ile}^{9}, \mathrm{Ser}^{11}$, Pro $^{16}$ while all the others contained Lys $^{2}$, Leu ${ }^{9}$, Tyr $^{11}$, $\mathrm{Ala}^{16}$. Seven peaks (peak 2, peak 3, peak 6, peak 12 to peak 15) were identified as $\mathrm{PLA}_{2} \mathrm{~s}$ and designated as $\mathrm{PLA}_{2} 1$ to $\mathrm{PLA}_{2} 7$ according to the order of elution. $\mathrm{PLA}_{2} 2, \mathrm{PLA}_{2} 5, \mathrm{PLA}_{2} 6$ and $\mathrm{PLA}_{2}$ of $N$. naja (India) (P15445) had the same partial sequences, including Lys ${ }^{10}$. On the other hand, partial amino acid sequences of $\mathrm{PLA}_{2} 1, \mathrm{PLA}_{2} 3, \mathrm{PLA}_{2} 4$ and $\mathrm{PLA}_{2} 7$ were novel for $N$. naja $\mathrm{PLA}_{2}$. Like $\mathrm{PLA}_{2} \mathrm{~s}$ of other elapid snakes, all the $\mathrm{PLA}_{2} \mathrm{~S}$ of the Indian cobra (Sri Lanka) belong to Group II with common Cys ${ }^{11}$. Peak 4 and peak 9 shared the same amino terminal sequence and were identified as MTLPs from their homology to MTLP-3 of N. kaouthia (P82464). These two MTLPs were the first of their type reported for N. naja as they contained $\mathrm{Ile}^{9}$ and $\mathrm{Arg}^{10}$ instead of $\mathrm{Arg}^{9}$ and $\mathrm{Thr}^{10}$, like those previously reported for $N$. atra and N. kaouthia. On the other hand, peak 5 was identified as a NGF because the amino-terminal 26 amino acid sequence was completely identical to those of $N$. naja (P01140), $N$. atra (P61898), N. sputatrix (Q5YF89) and N. kaouthia (A59218). Peak 7 and 8 were judged to be CRISPs, based on the fact that they shared identical amino-terminal 33 amino acid residues with those of Natrin-1 (Q7T1K6) of N. atra and Kaouthin-1 (ACH73167) of N. kaouthia, the proteins of which both belong to the CRISP family. A partial amino acid sequence (36 amino acid residues) of peak 1 showed the highest similarity $(30 / 36)$ to that of a thrombin-like serine protease of the Hardwicke's spine-bellied sea snake (Lapemis hardwickii). We then examined the thrombin-like activity in the elution on the SP column and found that only the first sharp peak in fraction 16 in Fig. 1B (left) showed strong hydorolytic activity towards Boc-Asp(oBzl)- 
Table 1 List of the amino-terminal amino acid sequences of the purified proteins from the venom of the Russell's viper (Sri Lanka) and those of related proteins

\begin{tabular}{|c|c|c|c|c|c|}
\hline Peak no/Ac no & Subspecies (Habitat) & \multicolumn{3}{|c|}{ N-terminal amino acid sequence } & Proposed name \\
\hline \multicolumn{6}{|c|}{$\mathrm{N}$ type phospholipase $\mathrm{A}_{2}\left(\mathrm{PLA}_{2}\right)$} \\
\hline Peak 6 & russellii (Sri Lanka) & NLFQFGEMIL & EKTGKEA & & $\mathrm{PLA}_{2} 1 *$ \\
\hline ABD24039 & russellii (India) & $\bullet \bullet \bullet A \bullet \bullet V$ & 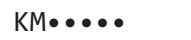 & & \\
\hline P81458 & russellii (India) & $\bullet \bullet \bullet A \bullet \bullet \vee V$ & $\mathrm{KM} \bullet \bullet N \mathrm{NP}$ & & \\
\hline ABD24037 & russellii (India) & $\bullet \bullet \bullet A \bullet \bullet D$ & $A \cdot Q E A F S$ & & \\
\hline P31100 & siamensis (Burma) & $\bullet \bullet \bullet \bullet \bullet \bullet \bullet$ & $\bullet \bullet \bullet \bullet V$ & & \\
\hline $2 \mathrm{H} 4 \mathrm{C} \mathrm{G}$ & siamensis (Burma) & $\bullet F \bullet \bullet A \bullet \bullet V$ & 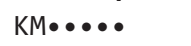 & & \\
\hline 1OQS A & siamensis (Burma) & $\bullet \bullet \bullet A R \bullet \bullet N$ & $G \bullet L \bullet A F S$ & & \\
\hline $2 \mathrm{H} 4 \mathrm{C} \mathrm{H}$ & siamensis (Burma) & $\bullet \bullet \bullet A R L \bullet D$ & $A \cdot Q E A F S$ & & \\
\hline AAP13805 & siamensis (Burma) & $\bullet Y \bullet \bullet \bullet \bullet N$ & $Q \bullet \bullet N F G$ & & \\
\hline ACD43469 & siamensis (Burma) & $\bullet \bullet \bullet \bullet \cdot \bullet \cdot N$ & $\mathrm{~K} \bullet \bullet \mathrm{RFG}$ & & \\
\hline 1OQS G & siamensis (Burma) & $\bullet \cdots \cdots \cdots \cdots \bullet$ & $Q \bullet \bullet \bullet \vee V$ & & \\
\hline AAZ53184 & siamensis (Java) & $\bullet \bullet \bullet \bullet \bullet \bullet \bullet$ & $\bullet \bullet \bullet \bullet V$ & & \\
\hline AAZ53185 & siamensis (Java) & $\bullet \bullet \bullet A R \bullet \bullet N$ & $G \bullet L \bullet A F S$ & & \\
\hline \multicolumn{6}{|c|}{$\mathrm{S}$ type phospholipase $\mathrm{A}_{2}\left(\mathrm{PLA}_{2}\right)$} \\
\hline Peak 9 & russellii (Sri Lanka) & SLLEFGMMIL & EETGKLAVPF & YSSY & $\mathrm{PLA}_{2} 2 *$ \\
\hline Peak 11 & russellii (Sri Lanka) & $\bullet \ldots \ldots \ldots \cdots$ & $\bullet \ldots \ldots \ldots$ & $\cdots \cdots$ & $\mathrm{PLA}_{2} 3 *$ \\
\hline Peak 12 & russellii (Sri Lanka) & $\bullet \bullet \bullet \bullet \bullet \bullet \bullet$ & $\bullet \bullet \bullet \bullet I \bullet S$ & • & $\mathrm{PLA}_{2} 4$ \\
\hline Peak 13 & russellii (Sri Lanka) & $\bullet \bullet \bullet \bullet \bullet \bullet \bullet$ & $\bullet \bullet \bullet \bullet \bullet \bullet \bullet$ & $\bullet \bullet \bullet$ & $\mathrm{PLA}_{2} 5^{*}$ \\
\hline $2 \mathrm{DO} 2 \mathrm{~A}$ & russellii (Sri Lanka) & $\bullet \bullet \bullet \bullet К \bullet \bullet$ & $\bullet \bullet \bullet \bullet I \bullet S$ & $\bullet \bullet \bullet$ & \\
\hline 2PVT A & russellii (Sri Lanka) & $\bullet I \bullet K \bullet \bullet$ & $\bullet \bullet \bullet \cdot \mathrm{I} \bullet S$ & $\bullet \bullet$ & \\
\hline 1OYF A & russellii (Sri Lanka) & $\bullet \bullet \bullet \bullet \bullet \bullet \bullet$ & $\bullet \bullet \bullet \bullet I \bullet S$ & $\bullet \bullet$ & \\
\hline P84674 & russellii (India) & $\bullet \bullet \bullet \bullet \bullet \bullet$ & $\bullet \bullet \bullet \bullet I \bullet S$ & $\bullet \bullet$ & \\
\hline 1TGM A & russellii (India) & $\bullet \bullet \bullet \bullet К \bullet \bullet$ & $\bullet \bullet \bullet \bullet I \bullet S$ & $\bullet \bullet \bullet$ & \\
\hline AAZ53186 & russellii (Java) & $\bullet \bullet \bullet \bullet \bullet \bullet \bullet$ & $\bullet \bullet \bullet \bullet I \bullet S$ & $\bullet \bullet \bullet$ & \\
\hline \multicolumn{6}{|c|}{ Russell's viper venom factor V activating enzyme (RVV-V) } \\
\hline Peak 1 & russellii (Sri Lanka) & VIGGDECNIN & EHPFLA & & RVV-V1* \\
\hline Peak 3 & russellii (Sri Lanka) & $\bullet V \bullet \bullet \bullet \bullet \bullet \bullet$ & $\bullet$ RS• & & $\mathrm{RVV}-\mathrm{V} 2 *$ \\
\hline Peak 4 & russellii (Sri Lanka) & $\bullet \vee \bullet \bullet \bullet \bullet \bullet \bullet$ & $\bullet$ RS • & & RVV-V3* \\
\hline Peak 10 & russellii (Sri Lanka) & $\bullet \vee \bullet \bullet \bullet \bullet \bullet \bullet$ & $\bullet \bullet \bullet \vee V$ & & RVV-V4 \\
\hline P18964 & siamensis (India) & $\bullet \vee \bullet \bullet \bullet \bullet \bullet \bullet$ & $\bullet \bullet \bullet \vee$ & & \\
\hline P18965 & siamensis (India) & $\bullet \vee \bullet \bullet \bullet \bullet \bullet \bullet$ & $\bullet \bullet \bullet \vee$ & & \\
\hline \multicolumn{6}{|c|}{ C-type lectin-like protein (CLP) } \\
\hline Peak 7-1 & russellii (Sri Lanka) & NQDCLSDWSF & YEQYCYKVF & & CLP1* \\
\hline Peak 7-2 & russellii (Sri Lanka) & $\bullet \bullet R P G \bullet \bullet$ & $\bullet \bullet N \bullet \cdots \bullet$ & & CLP2* \\
\hline AAY 63870 & siamensis (Burma) & $\mathrm{GL} \bullet P P \bullet S \bullet L$ & $\bullet$ RYF•••• & & \\
\hline AAY 63871 & siamensis (Burma) & $V L \bullet \bullet \bullet \cdot G \bullet L S$ & $\bullet \bullet H \bullet \bullet \bullet \bullet \bullet$ & & \\
\hline Q4PRC0 & siamensis (Burma) & $\mathrm{AFC} \bullet \mathrm{P} \bullet \mathrm{G} \bullet \bullet A$ & $\bullet D \bullet N \bullet \bullet \bullet \bullet$ & & \\
\hline Q4PRC9 & siamensis (Burma) & $\mathrm{AFC} \bullet \mathrm{P} \bullet \mathrm{G} \bullet \bullet \mathrm{A}$ & $\bullet D \bullet N \bullet \bullet \bullet \bullet$ & & \\
\hline Q4PRC8 & siamensis (Burma) & $K \bullet \cdots \cdots \cdots \bullet$ & $\bullet \bullet G \bullet \bullet \bullet \bullet \bullet$ & & \\
\hline Q4PRC7 & siamensis (Burma) & $\mathrm{GA} \bullet \bullet \cdot \mathrm{E} \bullet \bullet \mathrm{S}$ & $\mathrm{H} \bullet \mathrm{GH} \bullet \cdots \bullet$ & & \\
\hline Q4PRC6 & siamensis (China) & $K \bullet \bullet \cdots \cdots \bullet$ & 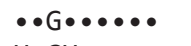 & & \\
\hline ABAY8656 & siamensis (Burma) & $\mathrm{GA} \bullet \bullet \cdot \mathrm{P} \bullet \bullet \mathrm{S}$ & $\mathrm{H} \bullet \mathrm{GH} \bullet \bullet \bullet \bullet$ & & \\
\hline \multicolumn{6}{|c|}{ L-amino acid oxidase (LAO) } \\
\hline Peak 5-1 & russellii (Sri Lanka) & ADDKNPLEEC & FREDDYEEF & & LAO ${ }^{*} *$ \\
\hline Peak 5-2 & russellii (Sri Lanka) & $\bullet \bullet \bullet \bullet \bullet \bullet \bullet$ & $\bullet \bullet \bullet \bullet \bullet \bullet$ & & LAO2* \\
\hline \multicolumn{6}{|c|}{ Russell's viper venom factor X-activating enzyme (RVV-X) } \\
\hline Peak 2 & russellii (Sri Lanka) & VATSEQFNKT & FIELVIVVD & & RVV-X* \\
\hline Q7LZ61 & russellii (India) & $\bullet S \bullet \cdot A \bullet \cdots I$ & $\bullet \bullet \bullet \bullet \cdot \bullet \bullet$ & & \\
\hline \multicolumn{6}{|c|}{ cysteine-rich secretory protein (CRISP) } \\
\hline Peak 8 & russellii (Sri Lanka) & SVDFDSESPR & & & CRISP* \\
\hline
\end{tabular}

* Indicates the sequence is unique for D. russellii in the protein database.

Peak numbers are identical with those of Fig. 1A.

Identification of isolated toxins is based on database sequence homology searching. 
Table 2 List of the amino-terminal amino acid sequences of the purified protein components from the venoms of Naja naja living in Sri Lanka and India

\begin{tabular}{|c|c|c|c|c|c|c|}
\hline Peak no/Ac no & Habitat & \multicolumn{4}{|c|}{ N-terminal amino acid sequence } & Proposed name \\
\hline \multicolumn{7}{|l|}{ cytotoxin (CTX) } \\
\hline Peak 10 & Sri Lanka & LQCNKLVPIA & SKTCPPGK & & & CTX1* \\
\hline Peak 11 & Sri Lanka & $\bullet \cdots \cdots \cdots \cdots$ & $\bullet \cdots \cdots \cdots \mathrm{NL}$ & CYKMFMVSD & & $\mathrm{CTX} 2 *$ \\
\hline Peak 16 & Sri Lanka & $\bullet K \bullet \bullet \bullet I \bullet L \bullet$ & $Y \bullet \bullet \bullet A \bullet \bullet N L$ & CYKMYMVATP & & CTX3* \\
\hline Peak 17 & Sri Lanka & $\bullet K \bullet \bullet \bullet I \bullet L \bullet$ & $Y \bullet \bullet \bullet A \bullet N L$ & $\mathrm{C}$ & & CTX4 \\
\hline Peak 18 & Sri Lanka & $\bullet K \bullet \bullet \bullet I \bullet L \bullet$ & $Y \bullet \bullet \bullet \bullet$ & & & CTX5 \\
\hline Peak 19 & Sri Lanka & $\bullet K \bullet \bullet \bullet \bullet \bullet L \bullet$ & $Y \bullet \bullet \bullet \bullet$ & & & CTX6 \\
\hline Peak 20 & Sri Lanka & $\bullet K \bullet \bullet \bullet I \bullet L \bullet$ & $Y \bullet \bullet \bullet A \bullet D L$ & CYKMYMVSNK & & CTX7* \\
\hline Peak 21 & Sri Lanka & $\bullet K \bullet \bullet \bullet I \bullet L \bullet$ & $Y \bullet \bullet \cdot A \bullet D L$ & CYKMYMVSDK & & CTX8* \\
\hline Peak 22 & Sri Lanka & $\bullet K \bullet \bullet \bullet \bullet \bullet L F$ & $\mathrm{Y} \bullet \bullet \bullet A \bullet \bullet N L$ & CYKMYMVATP & & CTX9 \\
\hline Peak 23 & Sri Lanka & $\bullet K \bullet \bullet \bullet \bullet \bullet L F$ & $Y \bullet \bullet \bullet A \bullet D L$ & CYKMYMVATP & & CTX10* \\
\hline P01447 & India & $\bullet K \bullet \bullet \bullet I \bullet L \bullet$ & $Y \bullet \cdots A \bullet N L$ & CYKMYMVSNK & & \\
\hline $\mathrm{P} 24780$ & India & $\bullet K \bullet \bullet \bullet \bullet L F$ & $Y \bullet \cdots A \bullet N L$ & CYKMFMVSNK & & \\
\hline P01440 & India & $\bullet K \bullet \bullet \bullet \bullet \bullet L F$ & $\mathrm{Y} \bullet \bullet \bullet A \bullet \bullet N L$ & CYKMYMVATP & & \\
\hline \multicolumn{7}{|c|}{ phospholipase $\mathrm{A}_{2}\left(\mathrm{PLA}_{2}\right)$} \\
\hline Peak 2 & Sri Lanka & NLYQFKNMIQ & $\mathrm{CT}$ & & & $\mathrm{PLA}_{2} 1 *$ \\
\hline Peak 3 & Sri Lanka & $\bullet \cdots \cdots \cdots K$ & $\bullet$ •VPSRSWWD & & & $\mathrm{PLA}_{2} 2$ \\
\hline Peak 6 & Sri Lanka & 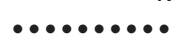 & $\bullet$.VPS & & & $\mathrm{PLA}_{2} 3 *$ \\
\hline Peak 12 & Sri Lanka & $\bullet \bullet \bullet \bullet \bullet \bullet \bullet \bullet$ & $\bullet \bullet V$ & & & $\mathrm{PLA}_{2} 4 *$ \\
\hline Peak 13 & Sri Lanka & $\bullet \bullet \bullet \bullet \bullet K$ & $\bullet$ & & & $\mathrm{PLA}_{2} 5$ \\
\hline Peak 14 & Sri Lanka & $\bullet \cdots \cdots \cdots \cdots K$ & $\bullet$ •VPSRSWWD & & & $\mathrm{PLA}_{2} 6$ \\
\hline Peak 15 & Sri Lanka & $\bullet \bullet \bullet \bullet \bullet \bullet \bullet \bullet$ & $\bullet$ & & & $\mathrm{PLA}_{2} 7 *$ \\
\hline $\mathrm{P} 15445$ & India & $\bullet \bullet \bullet \bullet \bullet \bullet$ & $\bullet \bullet V P S R S W W D$ & & & \\
\hline \multicolumn{7}{|c|}{ cystein-rich secretory protein (CRISP) } \\
\hline Peak 7 & Sri Lanka & NVDFNSESTR & RKKKQKEIVD & LHNSLRRRVS & PTA & CRISP1* \\
\hline Peak 8 & Sri Lanka & $\bullet \bullet \bullet \bullet \bullet \bullet$ & $\bullet$ & & & CRISP2* \\
\hline \multicolumn{7}{|c|}{ muscarinic toxin-like protein (MTLP) } \\
\hline Peak 4 & Sri Lanka & TICYNHLTIR & & & & MTLP1* \\
\hline Peak 9 & Sri Lanka & $\bullet \bullet \bullet \bullet \bullet \bullet \bullet$ & SEVTEICIIC & DDDYYF & & MTLP2* \\
\hline \multicolumn{7}{|c|}{ nerve growth factor (NGF) } \\
\hline Peak 5 & Sri Lanka & EDHPVHNLGE & HSVCDSVSAW & VTKTTA & & NGF \\
\hline P01140 & India & $\bullet \bullet \bullet \bullet \bullet \bullet \bullet$ & $\bullet \bullet \bullet \bullet \bullet \bullet \bullet$ & $\bullet \bullet \bullet \bullet$ & & \\
\hline \multicolumn{7}{|c|}{ thrombin-like serine protease (TLP) } \\
\hline Peak 1 & Sri Lanka & IGGFECNEHE & HRSLVYLYNS & AGFFCAGTLL & NHEWVV & TLP* \\
\hline
\end{tabular}

Pro-Arg-MCA, a synthetic substrate for thrombin. This fraction 16 was found to contain 2 kinds of proteins, peak 1 and peak 2 upon RP-HPLC (in the upper right of Fig. 1B) and the peak 2 was confirmed to be PLA 2 . Thus, we concluded that peak 1 was responsible for the thrombin-like protease activity.

Entire sequence of $P \mathrm{LA}_{2} 3$, the primary component of the venom of D. r. russellii

Many varieties were found in the amino-terminal amino acid sequences of the venom proteins of $D . r$. russellii and N. naja. To further clarify the variations in the amino acid sequences of the venom proteins, we determined the complete amino acid sequence of $\mathrm{PLA}_{2} 3$, the primary component of the venom. The entire amino acid sequence of $\mathrm{PLA}_{2} 3$ for D. r. russellii (Sri Lanka) is shown in Fig. 2A together with those of the $\mathrm{S}$ type $\mathrm{PLA}_{2}$ reported for D. r. russellii living in India and Sri Lanka. $\mathrm{PLA}_{2} 3$ with a molecular weight of 14,600 consists of 121 amino acids, including 14 Cys, 17 basic amino acids, and 14 acidic amino acids. All the 14 Cys residues are conservative in primary structure of the $\mathrm{S}$ 

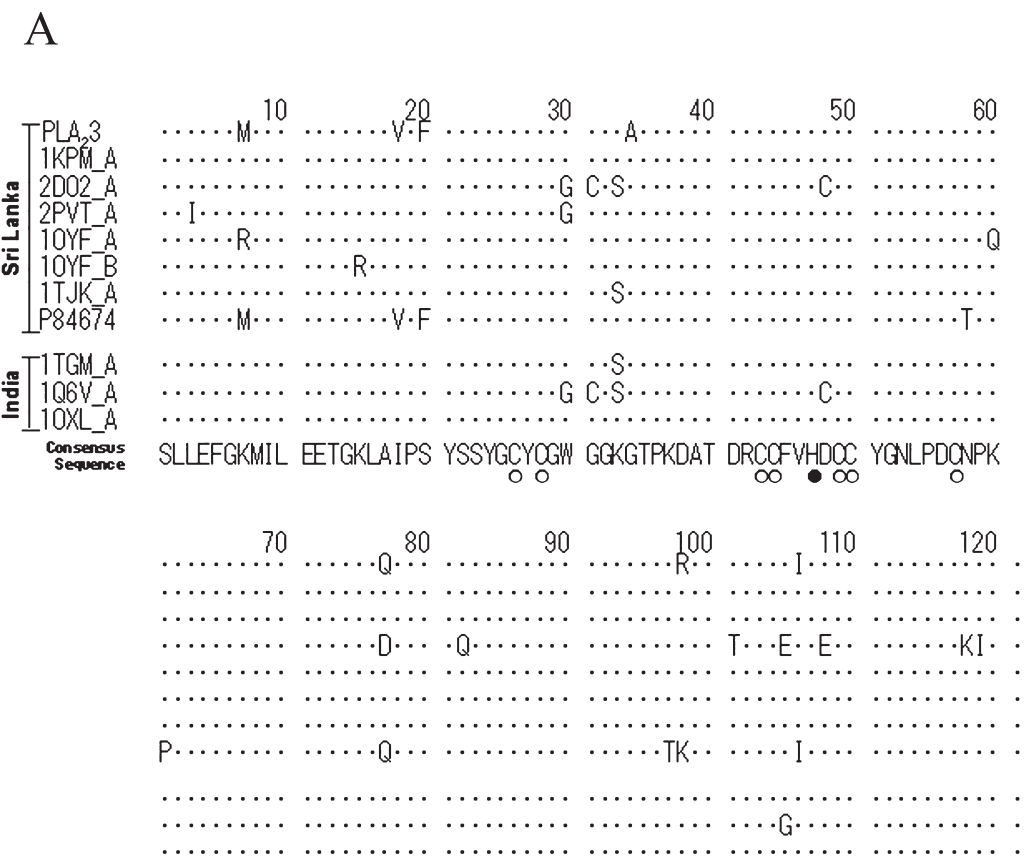

SDRYKYKRYN GAIVCEKGTS CENRICECOK ABAICFRONL NTYSKKYMLYY PDFLCKCELK C

$\mathrm{B}$

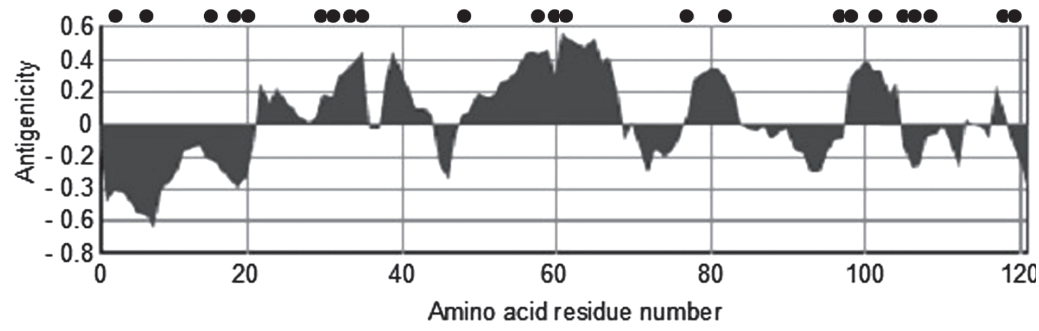

Fig. 2 Characteristic features of the primary sequence of $\mathrm{PLA}_{2} 3$ from $D$. $r$. russellii (Sri Lanka). (A) Amino acid sequence of $\mathrm{PLA}_{2} 3$ from D. r. russellii (Sri Lanka) with those of the $S$ type of PLA from D. r. russellii in Sri Lanka and India. The symbol $\bullet$ indicates the three catalytic residues and $\circ$ indicates the conserved Cys residues. (B) The predicted antigenicity. Analysis was carried out by a modified version of Jameson and Wolf algorithm (10) with a window width of 7 . The symbol $\bullet$ denotes the polymorphic amino acid residues found in $\mathrm{PLA}_{2} S$ of $D$. r. russellii. The accession numbers P86368 for PLA 3 is P86368 in the UniProt Knowledgebase.

type $\mathrm{PLA}_{2} \mathrm{~s}$ of $D$. r. russellii. $\mathrm{His}^{47}, \mathrm{Tyr}^{51}$ and $\mathrm{Asp}^{89}$, which are known to be the essential residues for the activity, were also conserved. An $\mathrm{Asp}^{48}$, providing $\mathrm{Ca}^{2+}$ binding site, was also found in this $\mathrm{PLA}_{2}$ and the $\mathrm{Ala}^{34}$ and $\mathrm{Arg}^{98}$ residues were unique to $\mathrm{PLA}_{2} 3$.

The positions where the amino acid variations are found among $\mathrm{PLA}_{2} \mathrm{~S}$ of $D$.r. russellii are indicated by solid circles in Fig. 2A. Out of 121 amino acid residues, variations were found in 23 positions. Interestingly, these variable amino acid residues were located on the potential antigenic sites as shown in Fig. 2B.
Entire sequence of $C T X 7$, the primary component of the venom of $N$. naja

To further clarify the variations in the amino acid sequence of the venom proteins, the complete amino acid sequence of CTX7 of $N$. naja, the primary component in the venom of $N$. naja, was also determined. As for CTXs of Indian cobra venom, only 3 sequences have been previously reported. The sequence of CTX7 of $N$. naja (Sri Lanka) was most similar to the CTX of $N$. naja in the Indian subcontinent (P01447) with differences in the position of 19, 48 and 49 (Fig. 3A). The CTX7 molecule seems 


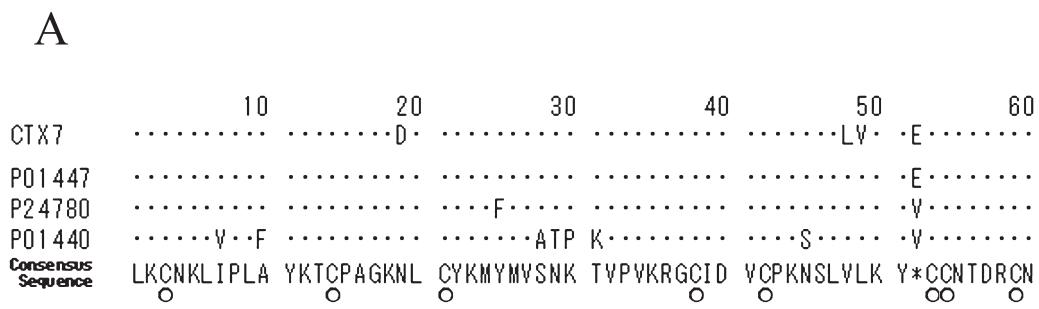

B

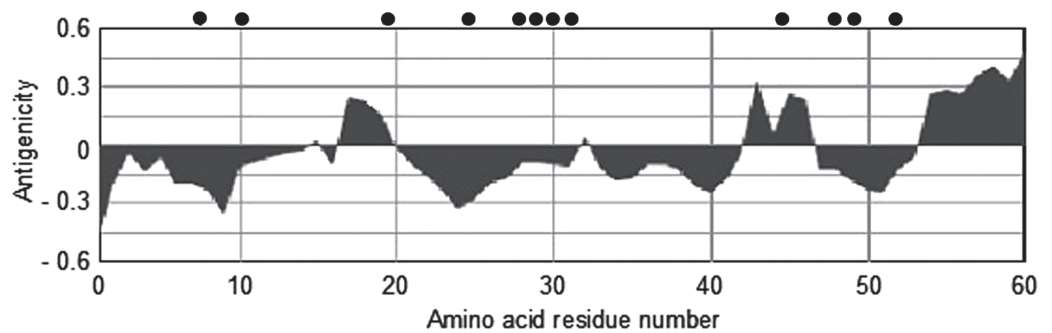

Fig. 3 Characteristic features of the primary sequence of CTX7 of $N$. naja (Sri Lanka). (A) Amino acid sequence of CTX7 of $N$. naja (Sri Lanka) with those of the corresponding species in India. The symbol $\circ$ indicates the conserved Cys residues. (B) The predicted antigenicity. The symbol • denotes the polymorphic amino acid residues found in CTX of N. naja (Sri Lanka). The accession number for CTX7 is P86382 in the UniProt Knowledgebase.

to have less antigenicity than the $\mathrm{PLA}_{2}$ molecule from D. russellii (Fig. $3 \mathrm{~A}$ and B). Both $\mathrm{Ala}^{34}$ and $\mathrm{Arg}^{98}$ residues, which were unique to CTX7 from $N$. naja (Sri Lanka), were located in the antigenic site.

Phylogenetic trees of the $S$ type of $P L A_{2}$ from $D$. russellii and CTX from $N$. naja

As shown in Fig. 4A, the phylogenetic tree of $\mathrm{PLA}_{2}$ indicates that $\mathrm{PLA}_{2}$ from $D$. russellii is classified into two types, the $\mathrm{N}$ type and the $\mathrm{S}$ type. At present, D. russellii is classified into two subspecies, namely D. r. russellii and D. r. siamensis. The former subspecies lives in the Indian subcontinent and in Sri Lanka, while the latter lives in Burma, Taiwan, and on Java. Four of five isozymes of PLA from $D$. r. russellii (Sri Lanka) are the $\mathrm{S}$ type, while both $\mathrm{N}$ and $\mathrm{S}$ types are found in the venom of $D . r$. russellii (India). Only $\mathrm{N}$ types of $\mathrm{PLA}_{2} \mathrm{~s}$ have been found in the venom of D.r. siamensis (Burma), while both $\mathrm{N}$ and $\mathrm{S}$ type have been found in the venom of $D$. $r$. siamensis on Java. In the protein database, there are only 3 amino acid sequences have been reported for the CTX of the Indian cobra in India subcontinent. The CTX7 of N. naja (Sri Lanka) is very similar to P01447 with only two homologous amino acid changes (Fig. 4B).

\section{DISCUSSION}

From the venom of the Russell's viper (Daboia russellii russellii) and the Indian cobra (Naja naja) both living in Sri Lanka, we purified 15 and 23 proteins, respectively. The amino-terminal amino acid sequence determination revealed that 13 proteins out of 15 and 15 proteins out of 23 exhibited new sequences for the corresponding snakes, despite the fact that only partial sequences were compared. Such a wide variation of amino acid sequences of venom proteins in a species has been observed for many toxic proteins. For example, $\mathrm{PLA}_{2}$ and CTX families are reported to have evolved by gene duplication followed by evolution under strong positive adaptive selection $(20,29)$. On the other hand, the 26 amino-terminal amino acid residues of NGF were completely identical with those of $N$. naja (India). Such high homology or identity seems rather natural for ordinary (nontoxic) proteins when compared among genera.

In addition to the accelerated evolution, differentiation of the species may partly contribute to the variations in the primary sequence of proteins. The Russell's viper and the Indian cobra living in Sri Lanka seem to be on their way to differentiation into new species from those living in India. In fact, both snakes in Sri Lanka are morphologically differ- 

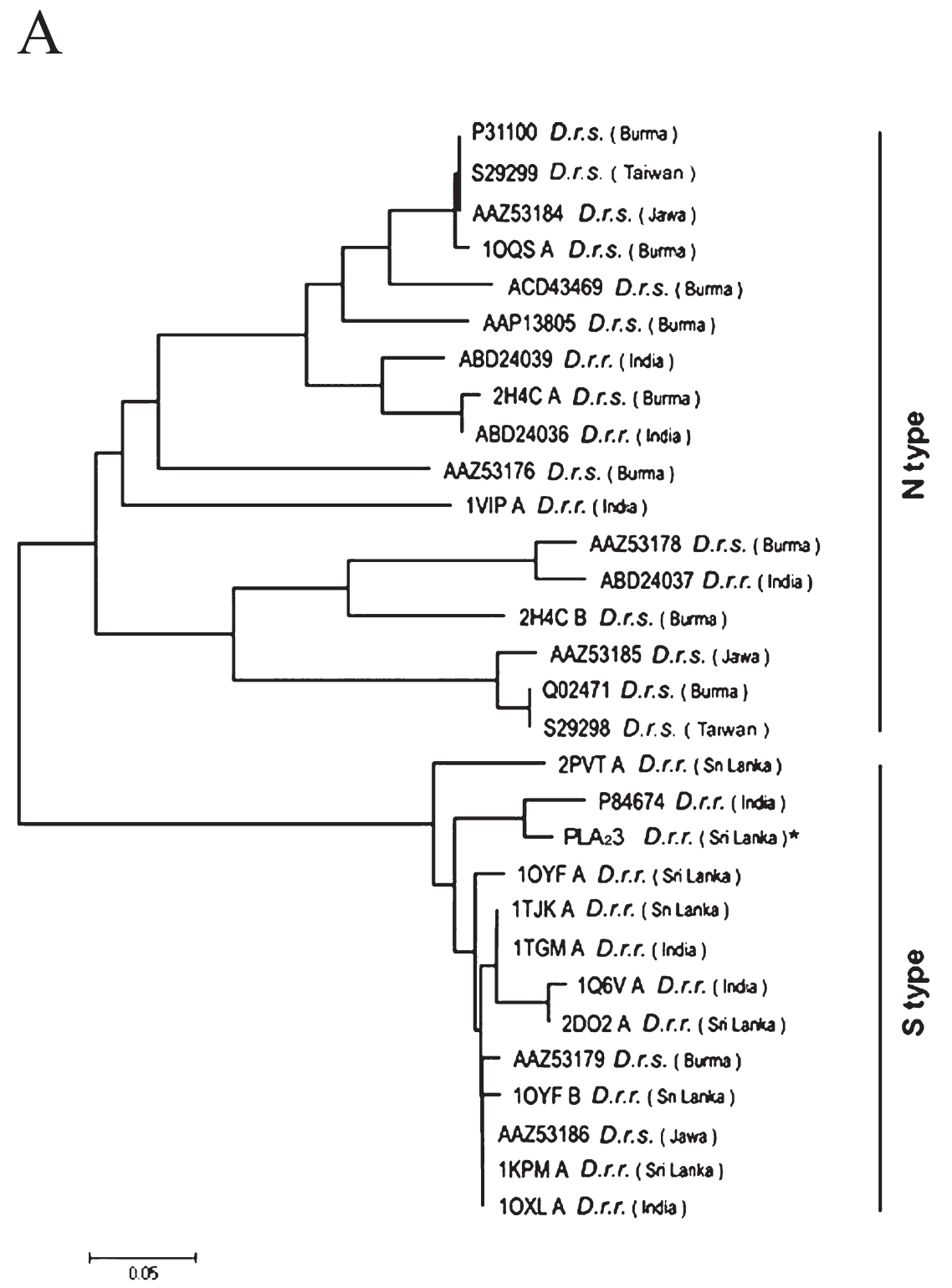

B
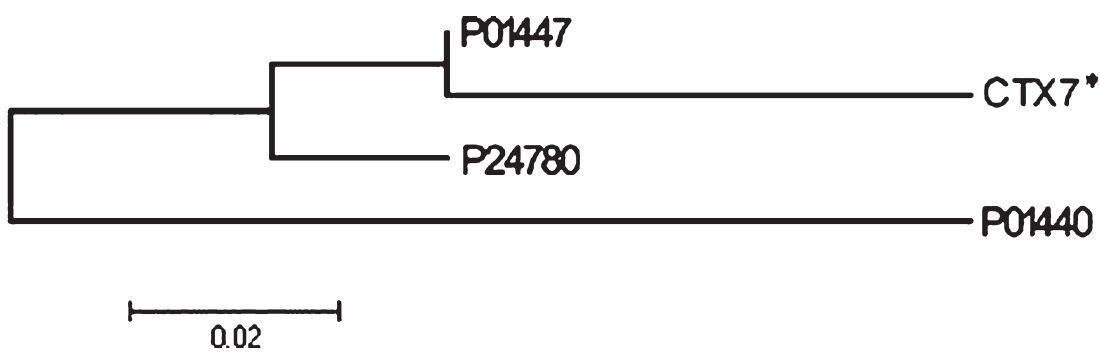

Fig. 4 Phylogenetic tree of $\mathrm{PLA}_{2}$ of $D$. russellii $(\mathrm{A})$ and CTX of $N$. naja (B). * The amino acid sequences of these proteins were determined in the present study. The NJ phylogenetic trees were drawn using the MEGA software program (23, 25). 
ent from those living in India and were previously classified as the endemic subspecies, D. $r$. pulchella and $N . n$. polyocellata. This nascent differentation is supported by a study which reports that in Sri Lanka several species of animals, including snakes, have been isolated from their counterparts in South India, though a land bridge between Sri Lanka and peninsular India has appeared and disappeared repeatedly over many thousands of years (1).

It is interesting that unique amino acid residues found in the S type of $\mathrm{PLA}_{2}$ from D. r. russellii (Sri Lanka) are preferentially located in potentially antigenic regions (Fig. 2B). These variations in the amino acid sequences may have resulted in the change of the antigenicity, which in turn may have resulted in the ineffectiveness of AVS.

Such is not clearly observed in the CTX of $N$. naja. It is interesting that Asn residue at the 20th position, which is unique to CTX7, is also located at the potential antigenic region of the CTX molecule. Accelerated evolution is thought to result in a family of toxins with a highly structural region containing Cys residues but widely varing functional activities. To improve the effectiveness of AVS, raising antiserum against the conserved sequence has seemed to be effective (9).

The $\mathrm{PLA}_{2} \mathrm{~S}$ of $D$. russellii are classified into two types, $\mathrm{N}$ type and $\mathrm{S}$ type, based on their respective primary sequences (27). Interestingly, the $\mathrm{PLA}_{2} \mathrm{~S}$ of D. r. russellii (Sri Lanka) were shown to be primarily $\mathrm{S}$ type and those of $D$. r. siamensis (Burma) were exclusively $\mathrm{N}$ type, whereas those of $D$. r. russellii (India) and D. r. siamensis (Java) were both $\mathrm{N}$ and $\mathrm{S}$ types. Similarly, it was also observed that toxin composition varied geographically in the subspecies of the Russell's viper in India (11). In addition to the variety of amino acid sequences of $\mathrm{PLA}_{2}$ isozymes, the relative content of $\mathrm{PLA}_{2}$ isozymes was different in $D$. russellii living in each region. This may not be the result of diversed PLA $\mathrm{P}_{2}$ but of an alteration of the expression of $\mathrm{PLA}_{2}$ genes as an adaptation for incapacitating prey, as has been previously suggested $(2,21)$.

This difference in expression patterns of $\mathrm{PLA}_{2}$ isozymes is certainly one of the causes of the ineffectiveness of local AVS. A telling example occurred when the antiserum against the $\mathrm{N}$ type PLA $\mathrm{A}_{2}$ from the Formosan $D$. $r$. siamensis did not react with the crude venom of the Sri Lankan D. r. russellii (16). The relative content of cytotoxins of $N$. atra is also known to be different in the eastern and western parts of India (9).

In conclusion, these present data suggest that variations in the amino acid sequences of each venom protein, which seem to be a result of accelerated evolution, and the geographical variation in the expression pattern of isotoxins have changed the antigenicity of snake venom at the local level, and consequently resulted in the ineffectiveness of universal AVS treatment methods.

\section{Acknowledgement}

This work was supported in part by the Heiwa Nakajima Foundation, Japan.

\section{REFERENCES}

1. Bossuy F, Meegaskumbura M, Beenaerts N, Gower DJ, Pethiyagoda R, Roelants K, Mannaert A, Wilkinson M, Bahir MM, Manamendra-Arachchi K, Ng PKL, Schneider CJ, Oommen, OV and Milinkovitch MC (2004) Local endemism within the Western Ghats-Sri Lanka biodiversity hotspot. Science 306, 479-481.

2. Daltry JC, Wuster W and Thorpe RS (1996) Diet and snake venom evolution. Nature 379, 537-540.

3. De Silva A and Ranasinghe L (1983) Epidemiology of snakebite in Sri Lanka. Ceylon Med J 28, 144-154.

4. De Silva HJ, Fonseka MM, Gunatilake SB, Kularatne SA and Sellahewa KH (2002) Anti-venom for snakebite in Sri Lanka. Ceylon Med J 47, 43-45.

5. Fox S, Rathuwithana AC, Kasturiratne A, Lalloo DG and De Silva HJ (2005) Underestimation of snakebite mortality by hospital statistics in the Monaragala district of Sri Lanka. Trans R Soc Trop Med Hyg 100, 693-695.

6. Friedman M (2001) Application of the S-pyridylethylation reaction to the elucidation of the structures and functions of proteins. J Protein Chem 20, 431-453.

7. Gibbs HL and Rossiter W (2008) Rapid evolution by positive selection and gene gain and loss: PLA $_{2}$ venom genes in closely related Sistrurus rattlesnakes with divergent diets. $J$ Mol Evol 66, 151-166.

8. Gong N, Armugam A and Jeyaseelan K (2000) Molecular cloning, characterization and evolution of the gene encoding a new group of short-chain $\alpha$-neurotoxins in an Australian elapid, Pseudonaja textilis. FEBS Lett 473, 303-310.

9. Harrison RA, Wüster W and Theakston RD (2003) The conserved structure of snake venom toxins confers extensive immunological cross-reactivity to toxin-specific antibody. Toxicon 41, 441-449.

10. Jameson BA and Wolf $H$ (1988) The antigenic index: a novel algorithm for predicting antigenic determinants. Comput Appl Biosci 4, 181-186.

11. Jayanthi GP and Gowda TV (1988) Geographical variation in India in the composition and lethal potency of Russell's viper (Vipera russelli) venom. Toxicon 26, 257-264.

12. Kaninaratline KE and Anandadas JA (1973) The use of antivenom in snake bite poisoning. Ceylon Med J 1, 37-43.

13. Kasturiratne A, Pathmeswaran A, Fonseka MM, Lalloo DG, Brooker S and De Silva HJ (2005) Estimates of disease burden due to land-snake bite in Sri Lankan hospitals. Southeast Asian J Trop Med Publ Health 36, 733-740.

14. Kasturiratne A, Wickremasinghe AR, De Silva N, Gunawardena NK, Pathmeswaran A, Premaratna R, Savioli L, Lalloo 
DG and De Silva HJ (2008) The global burden of snakebite: A literature analysis and modelling based on regional estimates of envenoming and deaths. PLoS Med 11, 1591-1604.

15. Kawabata S, Miura T, Morita T, Kato H, Fujikawa K, Iwanaga S, Takada K, Kimura T and Sakakibara S (1988) Highly sensitive peptide-4-methylcoumaryl-7-amide substrates for blood-clotting proteases and trypsin. Eur J Biochem 172, 17-25.

16. Kini RM and Chan YM (1999) Accelerated evolution and molecular surface of venom phospholipase $\mathrm{A}_{2}$ enzymes. $J$ Mol Evol 48, 125-132.

17. Kularatne SAM, Budagoda BDSS, Gawarammana IB and Kularatne WKS (2009) Epidemiology, clinical profile and management issues of cobra (Naja naja) bites in Sri Lanka: first authenticated case series. Trans $R$ Soc Trop Med Hyg 103, 924-930.

18. Mallow D, Ludwing D and Nilson G (2003) True Vipers: Natural History and Toxinology of Old World Vipers. Krieger Publ Co, Melbourne.

19. Nakashima K, Nobuhisa I, Deshimaru M, Nakai M, Ogawa T, Shimohigashi Y, Fukumaki Y, Hattori M, Sakai Y, Hattori S and Ohno M (1995) Accelerated evolution in the proteincoding regions is universal in crotalinae snake venom gland phospholipase A2 isozyme genes. Proc Natl Acad Sci USA 92, 5605-5609.

20. Ohno M, Menez R, Ogawa T, Danse JM, Shimohigashi Y, Fromen C, Ducancel F, Zinn-Justin S, Le Du MH, Boulain JC, Tamiya T and Menez A (1998) Molecular evolution of snake toxins: Is the functional diversity of snake toxins associated with a mechanism of accelerated evolution? Prog $\mathrm{Nu}$ cleic Acid Res Mol Biol 59, 307-364.

21. Pahari S, Bickford D, Fry BG and Kini RM (2007) Expression pattern of three-finger toxin and phospholipase A2 genes in the venom glands of two sea snakes, Lapemis curtus and
Acalyptophis peronii: Comparison of evolution of these toxins in land snakes, sea kraits and sea snakes. BMC Evolutionary Biology 7, 175-183.

22. Phillips RE, Theakston RD, Warrell DA, Galigedara Y, Abeysekera DT, Dissanayaka P, Hutton RA and Aloysius DJ (1988) Paralysis, rhabdomyolysis and haemolysis caused by bites of Russell's viper (Vipera russelli pulchella) in Sri Lanka: Failure of Indian (Haffkine) antivenom. $Q J \mathrm{Med} \mathbf{6 8}$, 691-715.

23. Saitou N and Nei M (1987) The neighbor-joining method: A new method for reconstructing phylogenetic trees. Mol Biol Evol 4, 406-425.

24. Sellahewa KH, Gunawardena G and Kumararatne MR (1995) Efficacy of antivenom in the treatment of severe local envenomation by the Hump-Nosed Viper (Hypnale hypnale). Am J Trop Med Hyg 53, 260-262.

25. Tamura K, Dudley J, Nei M and Kumar S (2007) MEGA4: Molecular evolutionary genetics analysis (MEGA) software ver 4.0. Mol Biol Evol 24,1596-1599.

26. Theakston RDG (1991) Immunological aspects of snake venom research. In: Handbook of Natural Toxins, Reptile and Amphibian Venoms, Vol. 5 (Tu A, ed.), pp 495-528, Marcel Dekker, New York.

27. Tsai IH, Lu PJ and Su JC (1996) Two types of Russell's viper revealed by variation in phospholipases $\mathrm{A}_{2}$ from venom of the subspecies. Toxicon 34, 99-109.

28. Woodhams BJ, Wilson SE, Xin BC and Hutton RA (1990) Differences between the venoms of two sub-species of Russell's viper: Vipera russelli pulchella and Vipera russelli siamensis. Toxicon 28, 427-433.

29. Wuster W (1996) Taxonomic changes and toxinology: Systematic revisions of the Asiatic cobras (Naja naja species complex). Toxicon 34, 399-406. 
